\title{
The existence of solutions for some fractional finite difference equations via sum boundary conditions
}

\author{
Ravi P Agarwal ${ }^{1,2}$, Dumitru Baleanu ${ }^{3,4^{*}}$, Shahram Rezapour ${ }^{5}$ and Saeid Salehi ${ }^{5}$
}

\section{"Correspondence:}

dumitru@cankaya.edu.tr

${ }^{3}$ Department of Mathematics,

Cankaya University, Ogretmenler

Cad. 14, Balgat, Ankara, 06530,

Turkey

${ }^{4}$ Institute of Space Sciences,

Magurele, Bucharest, Romania

Full list of author information is

available at the end of the article

\begin{abstract}
In this manuscript we investigate the existence of the fractional finite difference equation (FFDE) $\Delta_{\mu-2}^{\mu} x(t)=g(t+\mu-1, x(t+\mu-1), \Delta x(t+\mu-1))$ via the boundary condition $x(\mu-2)=0$ and the sum boundary condition $x(\mu+b+1)=\sum_{k=\mu-1}^{\alpha} x(k)$ for order $1<\mu \leq 2$, where $g: \mathbb{N}_{\mu-1}^{\mu+b+1} \times \mathbb{R} \times \mathbb{R} \rightarrow \mathbb{R}, \alpha \in \mathbb{N}_{\mu-1}^{\mu+b}$, and $t \in \mathbb{N}_{0}^{b+2}$. Along the same lines, we discuss the existence of the solutions for the following FFDE: $\Delta_{\mu-3}^{\mu} x(t)=g(t+\mu-2, x(t+\mu-2))$ via the boundary conditions $x(\mu-3)=0$ and $x(\mu+b+1)=0$ and the sum boundary condition $x(\alpha)=\sum_{k=\gamma}^{\beta} x(k)$ for order $2<\mu \leq 3$, where $g: \mathbb{N}_{\mu-2}^{\mu+b+1} \times \mathbb{R} \rightarrow \mathbb{R}, b \in \mathbb{N}_{0}, t \in \mathbb{N}_{0}^{b+3}$, and $\alpha, \beta, \gamma \in \mathbb{N}_{\mu-2}^{\mu+b}$ with $\gamma<\beta<\alpha$. MSC: $34 \mathrm{~A} 08$
\end{abstract}

Keywords: fractional finite difference equation; fixed point

\section{Introduction}

By the late 19th century, combined efforts made by several mathematicians led to a fairly solid understanding of fractional calculus in the continuous setting but significantly less is still known about discrete fractional calculus (see for example [1, 2] and [3] and the references therein). Recently, there has been a strong interest in this subject but still little progress was made in developing the theory of fractional finite difference equations (see [4-11] and [12] and the references therein).

Discrete fractional calculus is a powerful tool for the processes which appears in nature, e.g. biology, ecology and other areas (see for example [13] and [14] and the references therein), where the discrete models have to be considered in order to describe properly the complexity of the dynamical processes with memory effect. We notice that the existence of solutions for fractional finite difference equations is a hot topic of the fractional calculus with direct implications in modeling of some real world phenomena which have only discrete behaviors.

Motivated by the above mentioned results, in this paper we investigate the fractional finite difference equation

$$
\Delta_{\mu-2}^{\mu} x(t)=g(t+\mu-1, x(t+\mu-1), \Delta x(t+\mu-1))
$$

(02014 Agarwal et al.; licensee Springer. This is an Open Access article distributed under the terms of the Creative Commons Attribution License (http://creativecommons.org/licenses/by/2.0), which permits unrestricted use, distribution, and reproduction in any medium, provided the original work is properly cited. 
via the boundary conditions $x(\mu-2)=0$ and $x(\mu+b+1)=\sum_{k=\mu-1}^{\alpha} x(k)$, where $b \in \mathbb{N}_{0}$, $t \in \mathbb{N}_{0}^{b+2}, 1<\mu \leq 2, g: \mathbb{N}_{\mu-1}^{b+\mu+1} \times \mathbb{R} \times \mathbb{R} \rightarrow \mathbb{R}$, and $\alpha \in \mathbb{N}_{\mu-1}^{b+\mu}$.

Moreover, we investigate the FFDE given by

$$
\Delta_{\mu-3}^{\mu} x(t)=g(t+\mu-2, x(t+\mu-2))
$$

via the boundary conditions $x(\mu-3)=0, x(\mu+b+1)=0$, and $x(\alpha)=\sum_{k=\gamma}^{\beta} x(k)$, where $b \in \mathbb{N}_{0}, t \in \mathbb{N}_{0}^{b+3}, 2<\mu \leq 3, g: \mathbb{N}_{\mu-2}^{\mu+b+1} \times \mathbb{R} \rightarrow \mathbb{R}$ and $\alpha, \beta, \gamma \in \mathbb{N}_{\mu-2}^{\mu+b}$ with $\gamma<\beta<\alpha$.

In the following we present the basic definitions and theorems used in this manuscript. In Section 3 we present the main result. The manuscript ends with our conclusions.

\section{Preliminaries}

As you know, the gamma function is defined by

$$
\Gamma(z)=\int_{0}^{\infty} e^{-t} t^{z-1} d t
$$

which converges in the right half of the complex plane $\operatorname{Re}(z)>0$. It is well known that $\Gamma(z+1)=z \Gamma(z)$ and $\Gamma(n)=(n-1)$ ! for all $n \in \mathbb{N}$. Now, we define

$$
t^{\underline{\mu}}:=\frac{\Gamma(t+1)}{\Gamma(t+1-\mu)}
$$

for all $t, \mu \in \mathbb{R}$ [15]. If $t+1-\mu$ is a pole of the gamma function and $t+1$ is not a pole, then we define $t^{\underline{\mu}}=0$ [16]. For example, we have $(\mu-2) \stackrel{\mu-1}{=}=0$. Also, one can verify that $\mu^{\underline{\mu}}=\mu \frac{\mu-1}{\underline{\mu}}=\Gamma(\mu+1)$ and $\frac{t^{\underline{\mu+1}}}{t^{\underline{\mu}}}=t-\mu$.

In this paper, we use the notations $\mathbb{N}_{p}=\{p, p+1, p+2, \ldots\}$ for all $p \in \mathbb{R}$ and $\mathbb{N}_{p}^{q}=\{p, p+$ $1, p+2, \ldots, q\}$ for all real numbers $p$ and $q$ whenever $q-p$ is a natural number.

Let $\mu>0$ with $m-1<\mu<m$ for some natural number $m$. The $\mu$ th fractional sum of $f$ based at $a$ is defined as [3]

$$
\Delta_{a}^{-\mu} f(t)=\frac{1}{\Gamma(\mu)} \sum_{r=a}^{t-\mu}(t-\sigma(r))^{\frac{\mu-1}{}} f(r)
$$

for all $t \in \mathbb{N}_{a+\mu}$, where $\sigma(r)=r+1$ is the forward jump operator. Similarly, we define

$$
\Delta_{a}^{\mu} f(t)=\frac{1}{\Gamma(-\mu)} \sum_{r=a}^{t+\mu}(t-\sigma(r)) \frac{-\mu-1}{f} f(r)
$$

for all $t \in \mathbb{N}_{a+m-\mu}$ [17]. Note that the domain of $\Delta_{a}^{r} f$ is $\mathbb{N}_{a+m-r}$ for $r>0$ and $\mathbb{N}_{a-r}$ for $r<0$. Also, for the natural number $\mu=m$, we have the known formula [16]

$$
\Delta_{a}^{\mu} f(t)=\Delta^{m} f(t)=\sum_{i=0}^{m}(-1)^{i}\left(\begin{array}{c}
m \\
i
\end{array}\right) f(t+m-i)
$$

We define $\Delta_{a}^{0} f(t)=f(t)$ for all $t \in \mathbb{N}_{a}$, too. 
Lemma 2.1 [16] Let $g: \mathbb{N}_{a} \rightarrow \mathbb{R}$ be a mapping and $m$ a natural number. Then the general solution of the equation $\Delta_{a+\mu-m}^{\mu} x(t)=g(t)$ is given by

$$
x(t)=\sum_{i=1}^{m} C_{i}(t-a) \frac{\mu-i}{+}+\Delta_{a}^{-\mu} g(t)
$$

for all $t \in \mathbb{N}_{a+\mu-m}$, where $C_{1}, \ldots, C_{m}$ are arbitrary constants.

Let $g: \mathbb{N}_{\mu-1}^{b+1+\mu} \times \mathbb{R} \times \mathbb{R} \rightarrow \mathbb{R}$ be a mapping and $m$ a natural number. By using a similar proof, one can check that the general solution of the equation $\Delta_{\mu-m}^{\mu} x(t)=g(t+\mu-m+$ $1, x(t+\mu-m+1), \Delta x(t+\mu-m+1))$ is given by

$$
x(t)=\sum_{i=1}^{m} C_{i} t^{\mu-i}+\Delta^{-\mu} g(t+\mu-m+1, x(t+\mu-m+1), \Delta x(t+\mu-m+1))
$$

for all $t \in \mathbb{N}_{\mu-m}$. In particular, the general solution has the following representation:

$$
\begin{aligned}
x(t)= & \sum_{i=1}^{m} C_{i} t \frac{\mu-i}{\Gamma}+\frac{1}{\Gamma(\mu)} \sum_{r=0}^{t-\mu}(t-\sigma(r))^{\underline{\mu-1}} \\
& \times g(r+\mu-m+1, x(r+\mu-m+1), \Delta x(r+\mu-m+1))
\end{aligned}
$$

for all $t \in \mathbb{N}_{\mu-m}$. The next theorem plays an important role in our main results.

Theorem 2.2 [18] Every continuous function from a compact, convex, nonempty subset of a Banach space to itself has a fixed point.

\section{Main results}

In the following, we are ready to provide the main results. First, we investigate the FFDE

$$
\Delta_{\mu-2}^{\mu} x(t)=g(t+\mu-1, x(t+\mu-1), \Delta x(t+\mu-1))
$$

via the boundary conditions $x(\mu-2)=0$ and $x(\mu+b+1)=\sum_{k=\mu-1}^{\alpha} x(k)$, where $b \in \mathbb{N}_{0}$, $t \in \mathbb{N}_{0}^{b+2}, 1<\mu \leq 2, g: \mathbb{N}_{\mu-1}^{b+\mu+1} \times \mathbb{R} \times \mathbb{R} \rightarrow \mathbb{R}$, and $\alpha \in \mathbb{N}_{\mu-1}^{b+\mu}$.

Lemma 3.1 Let $b \in \mathbb{N}_{0}, t \in \mathbb{N}_{0}^{b+2}, 1<\mu \leq 2, g: \mathbb{N}_{\mu-1}^{b+\mu+1} \times \mathbb{R} \times \mathbb{R} \rightarrow \mathbb{R}$, and $\alpha \in \mathbb{N}_{\mu-1}^{b+\mu}$. Then $x_{0}$ is a solution of the problem

$$
\Delta_{\mu-2}^{\mu} x(t)=g(t+\mu-1, x(t+\mu-1), \Delta x(t+\mu-1))
$$

via the boundary conditions $x(\mu+b+1)=\sum_{k=\mu-1}^{\alpha} x(k)$ and $x(\mu-2)=0$ if and only if $x_{0}$ is a solution of the fractional sum equation

$$
x(t)=\sum_{r=0}^{b+1} G(t, r, \alpha) g(r+\mu-1, x(r+\mu-1), \Delta x(r+\mu-1)),
$$


where

$$
\begin{aligned}
G(t, r, \alpha)= & \frac{t^{\mu-1} \sum_{k=r+\mu}^{\alpha}(k-\sigma(r))^{\mu-1}}{\left((\mu+b+1) \frac{\mu-1}{-}-\frac{1}{\mu}(\alpha+1) \underline{\mu}\right) \Gamma(\mu)} \\
& -\frac{(\mu+b+1-\sigma(r)) \frac{\mu-1}{\mu} \frac{1}{\mu}(\alpha+1)^{\underline{\mu}} t^{\underline{\mu-1}}}{(\mu+b+1)^{\frac{\mu-1}{}} \Gamma(\mu)\left((\mu+b+1)^{\mu-1}-\frac{1}{\mu}(\alpha+1)^{\mu}\right)} \\
& -\frac{(\mu+b+1-\sigma(r)) \frac{\mu-1}{\mu} \frac{\mu-1}{}}{\Gamma(\mu)(\mu+b+1)^{\frac{\mu-1}{}}}+\frac{(t-\sigma(r)) \frac{\mu-1}{}}{\Gamma(\mu)}
\end{aligned}
$$

whenever $r<t-\mu \leq \alpha-\mu$ or $r \leq \alpha-\mu<t-\mu$,

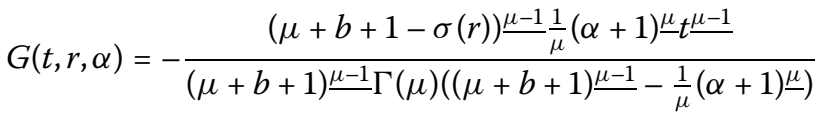

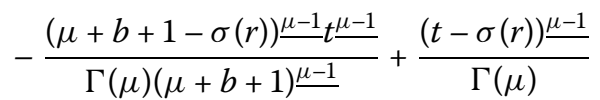

whenever $\alpha-\mu<r \leq t-\mu$,

$$
\begin{aligned}
G(t, r, \alpha)= & \frac{t \frac{\mu-1}{(} \sum_{k=r+\mu}^{\alpha}(k-\sigma(r))^{\mu-1}}{\left.(\mu+b+1)^{\frac{\mu-1}{}}-\frac{1}{\mu}(\alpha+1) \underline{\mu}\right) \Gamma(\mu)} \\
& -\frac{(\mu+b+1-\sigma(r)) \frac{\mu-1}{\mu} \frac{1}{\mu}(\alpha+1)^{\underline{\mu}} t^{\underline{\mu-1}}}{(\mu+b+1)^{\frac{\mu-1}{}} \Gamma(\mu)\left((\mu+b+1)^{\mu-1}-\frac{1}{\mu}(\alpha+1)^{\underline{\mu}}\right)} \\
& -\frac{(\mu+b+1-\sigma(r))^{\mu-1} t^{\mu-1}}{\Gamma(\mu)(\mu+b+1)^{\frac{\mu-1}{}}}
\end{aligned}
$$

whenever $t-\mu \leq r \leq \alpha-\mu$ and

$$
\begin{aligned}
G(t, r, \alpha)= & -\frac{(\mu+b+1-\sigma(r))^{\mu-1} \frac{1}{\mu}(\alpha+1)^{\mu} t \frac{\mu-1}{(\mu+b+1)^{\frac{\mu-1}{}} \Gamma(\mu)\left((\mu+b+1)^{\frac{\mu-1}{}}-\frac{1}{\mu}(\alpha+1)^{\underline{\mu}}\right)}}{} \\
& -\frac{(\mu+b+1-\sigma(r))^{\frac{\mu-1}{}} t^{\frac{\mu-1}{}}}{\Gamma(\mu)(\mu+b+1) \frac{\mu-1}{}}
\end{aligned}
$$

whenever $t-\mu \leq \alpha-\mu<r$ or $\alpha-\mu \leq t-\mu<r$.

Proof Let $x_{0}$ be a solution of the problem

$$
\Delta_{\mu-2}^{\mu} x(t)=g(t+\mu-1, x(t+\mu-1), \Delta x(t+\mu-1))
$$

via the boundary conditions $x(\mu+b+1)=\sum_{k=\mu-1}^{\alpha} x(k)$ and $x(\mu-2)=0$. By using Lemma 2.1, we get

$$
\begin{aligned}
x_{0}(t)= & C_{1} t \frac{\mu-1}{}+C_{2} t \frac{\mu-2}{2}+\Delta^{-\mu} g\left(t+\mu-1, x_{0}(t+\mu-1), \Delta x_{0}(t+\mu-1)\right) \\
= & C_{1} t \frac{\mu-1}{}+C_{2} t \frac{\mu-2}{\Gamma(\mu)}+\frac{1}{\Gamma=0} \sum_{r=0}^{t-\mu}(t-\sigma(r)) \frac{\mu-1}{} \\
& \times g\left(r+\mu-1, x_{0}(r+\mu-1), \Delta x_{0}(r+\mu-1)\right) .
\end{aligned}
$$


Since $x_{0}(\mu-2)=0$, we have

$$
\begin{aligned}
0= & C_{1}(\mu-2) \frac{\mu-1}{+}+C_{2}(\mu-2) \frac{\mu-2}{}+\frac{1}{\Gamma(\mu)} \sum_{r=0}^{(\mu-2)-\mu}((\mu-2)-\sigma(r))^{\frac{\mu-1}{}} \\
& \times g\left(r+\mu-1, x_{0}(r+\mu-1), \Delta x_{0}(r+\mu-1)\right) .
\end{aligned}
$$

Since $(\mu-2) \frac{\mu-1}{=}=0$ and

$$
\sum_{r=0}^{-2}((\mu-2)-\sigma(r))^{\frac{\mu-1}{g}} g\left(r+\mu-1, x_{0}(r+\mu-1), \Delta x_{0}(r+\mu-1)\right)=0
$$

$C_{2}=0$. On the other hand, we have $x_{0}(\mu+b+1)=\sum_{k=\mu-1}^{\alpha} x_{0}(k)$. Thus,

$$
\begin{aligned}
\sum_{k=\mu-1}^{\alpha} x_{0}(k)= & C_{1}(\mu+b+1) \frac{\mu-1}{+}+\frac{1}{\Gamma(\mu)} \sum_{r=0}^{b+1}(\mu+b+1-\sigma(r))^{\frac{\mu-1}{}} \\
& \times g\left(r+\mu-1, x_{0}(r+\mu-1), \Delta x_{0}(r+\mu-1)\right) .
\end{aligned}
$$

Hence,

$$
\begin{aligned}
C_{1}= & \frac{1}{(\mu+b+1) \frac{\mu-1}{2}}\left[\sum_{k=\mu-1}^{\alpha} x_{0}(k)-\frac{1}{\Gamma(\mu)} \sum_{r=0}^{b+1}(\mu+b+1-\sigma(r))^{\underline{\mu-1}}\right. \\
& \left.\times g\left(r+\mu-1, x_{0}(r+\mu-1), \Delta x_{0}(r+\mu-1)\right)\right]
\end{aligned}
$$

and so

$$
\begin{aligned}
x_{0}(t)= & \frac{1}{(\mu+b+1)^{\frac{\mu-1}{}}}\left[\sum_{k=\mu-1}^{\alpha} x_{0}(k)\right. \\
& \left.-\frac{1}{\Gamma(\mu)} \sum_{r=0}^{b+1}(\mu+b+1-\sigma(r))^{\frac{\mu-1}{}} g\left(r+\mu-1, x_{0}(r+\mu-1), \Delta x_{0}(r+\mu-1)\right)\right] t \underline{\mu-1} \\
& +\frac{1}{\Gamma(\mu)} \sum_{r=0}^{t-\mu}(t-\sigma(r))^{\frac{\mu-1}{g}} g\left(r+\mu-1, x_{0}(r+\mu-1), \Delta x_{0}(r+\mu-1)\right) .
\end{aligned}
$$

To calculate $\sum_{k=\mu-1}^{\alpha} x_{0}(k)$, taking the summation $\sum_{k=\mu-1}^{\alpha}$ on both sides of the above relation gives us

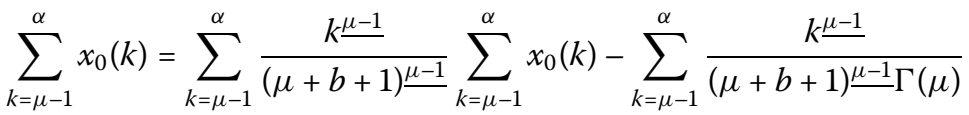

$$
\begin{aligned}
& \times \sum_{r=0}^{b+1}(\mu+b+1-\sigma(r))^{\frac{\mu-1}{}} g\left(r+\mu-1, x_{0}(r+\mu-1), \Delta x_{0}(r+\mu-1)\right) \\
& +\sum_{k=\mu-1}^{\alpha} \frac{1}{\Gamma(\mu)} \sum_{r=0}^{k-\mu}(k-\sigma(r))^{\frac{\mu-1}{g}} g\left(r+\mu-1, x_{0}(r+\mu-1), \Delta x_{0}(r+\mu-1)\right) \text {. }
\end{aligned}
$$


Hence,

$$
\begin{aligned}
& \sum_{k=\mu-1}^{\alpha} x_{0}(k)\left[1-\frac{\left.\sum_{k=\mu-1}^{\alpha} k \frac{\mu-1}{(\mu+b+1) \frac{\mu-1}{}}\right]}{(\mu+)^{\prime}}\right] \\
& \quad-\frac{\sum_{k=\mu-1}^{\alpha} k^{\mu-1}}{(\mu+b+1) \frac{\mu-1}{\Gamma} \Gamma(\mu)} \sum_{r=0}^{b+1}(\mu+b+1-\sigma(r))^{\mu-1} \\
& \quad \times g\left(r+\mu-1, x_{0}(r+\mu-1), \Delta x_{0}(r+\mu-1)\right) \\
& \quad+\sum_{k=\mu-1}^{\alpha} \frac{1}{\Gamma(\mu)} \sum_{r=0}^{k-\mu}(k-\sigma(r))^{\frac{\mu-1}{g}} g\left(r+\mu-1, x_{0}(r+\mu-1), \Delta x_{0}(r+\mu-1)\right),
\end{aligned}
$$

and so by interchanging the order of summations, we have

$$
\begin{aligned}
& \sum_{k=\mu-1}^{\alpha} x_{0}(k)=\sum_{r=0}^{\alpha-\mu} \frac{\sum_{k=r+\mu}^{\alpha} \frac{(k-\sigma(r))^{\mu-1}}{\Gamma(\mu)}}{1-\frac{\sum_{k=\mu-1}^{\alpha} k^{\mu-1}}{(\mu+b+1) \underline{\mu-1}}} g\left(r+\mu-1, x_{0}(r+\mu-1), \Delta x_{0}(r+\mu-1)\right)
\end{aligned}
$$

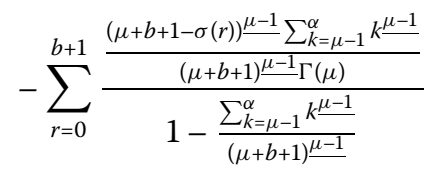

$$
\begin{aligned}
& \times g\left(r+\mu-1, x_{0}(r+\mu-1), \Delta x_{0}(r+\mu-1)\right) \text {. }
\end{aligned}
$$

Since

$$
\sum_{k=\mu-1}^{\alpha} k^{\mu-1}=\sum_{k=\mu-1}^{\alpha} \Delta_{k}\left(\frac{k^{\underline{\mu}}}{\mu}\right)=\frac{1}{\mu}\left((\alpha+1) \underline{\mu}-(\mu-1)^{\underline{\mu}}\right)=\frac{1}{\mu}(\alpha+1)^{\underline{\mu}},
$$

by replacing (3.3) in (3.1), we get

$$
\begin{aligned}
& x_{0}(t)=\sum_{r=0}^{\alpha-\mu} \frac{t \frac{\mu-1}{\sum_{k=r+\mu}^{\alpha}}(k-\sigma(r))^{\frac{\mu-1}{}}}{\left((\mu+b+1)^{\frac{\mu-1}{-}}-\frac{1}{\mu}(\alpha+1)^{\underline{\mu}}\right) \Gamma(\mu)} g\left(r+\mu-1, x_{0}(r+\mu-1), \Delta x_{0}(r+\mu-1)\right)
\end{aligned}
$$

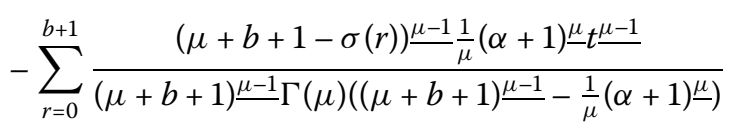

$$
\begin{aligned}
& \times g\left(r+\mu-1, x_{0}(r+\mu-1), \Delta x_{0}(r+\mu-1)\right) \\
& -\sum_{r=0}^{b+1} \frac{(\mu+b+1-\sigma(r)) \frac{\mu-1}{\mu} t^{\mu-1}}{\Gamma(\mu)(\mu+b+1) \frac{\mu-1}{}} g\left(r+\mu-1, x_{0}(r+\mu-1), \Delta x_{0}(r+\mu-1)\right) \\
& +\sum_{r=0}^{t-\mu} \frac{(t-\sigma(r)) \frac{\mu-1}{\Gamma}}{\Gamma(\mu)} g\left(r+\mu-1, x_{0}(r+\mu-1), \Delta x_{0}(r+\mu-1)\right) \\
& =\sum_{r=0}^{b+1} G(t, r, \alpha) g\left(r+\mu-1, x_{0}(r+\mu-1), \Delta x_{0}(r+\mu-1)\right) \text {. }
\end{aligned}
$$

Now, let $x_{0}$ be a solution of the fractional sum equation

$$
x(t)=\sum_{r=0}^{b+1} G(t, r, \alpha) g(r+\mu-1, x(r+\mu-1), \Delta x(r+\mu-1)) .
$$


Then $x_{0}$ is a solution of the equation

$$
\begin{aligned}
x(t)= & \frac{1}{(\mu+b+1) \frac{\mu-1}{2}}\left[\sum_{k=\mu-1}^{\alpha} x(k)\right. \\
& \left.-\frac{1}{\Gamma(\mu)} \sum_{r=0}^{b+1}(\mu+b+1-\sigma(r))^{\mu-1} g(r+\mu-1, x(r+\mu-1), \Delta x(r+\mu-1))\right] t \frac{\mu-1}{t-\mu} \\
& +\frac{1}{\Gamma(\mu)} \sum_{r=0}^{t-}(t-\sigma(r))^{\frac{\mu-1}{} g(r+\mu-1, x(r+\mu-1), \Delta x(r+\mu-1))} \\
= & \frac{1}{(\mu+b+1) \underline{\mu-1}}\left[\sum_{k=\mu-1}^{\alpha} x(k)-\frac{1}{\Gamma(\mu)} \sum_{r=0}^{b+1}(\mu+b+1-\sigma(r))^{\underline{\mu-1}}\right. \\
& \times g(r+\mu-1, x(r+\mu-1), \Delta x(r+\mu-1))] t \underline{\mu-1} \\
& +\Delta^{-\mu} g(t+\mu-1, x(t+\mu-1), \Delta x(t+\mu-1)) .
\end{aligned}
$$

It is easy to check that $x_{0}(\mu-2)=0$. Also, we have

$$
\begin{aligned}
x_{0}(\mu+b+1) & \\
= & \frac{1}{(\mu+b+1) \frac{\mu-1}{}}\left[\sum_{k=\mu-1}^{\alpha} x_{0}(k)-\frac{1}{\Gamma(\mu)} \sum_{r=0}^{b+1}(\mu+b+1-\sigma(r)) \frac{\mu-1}{}\right. \\
& \left.\quad \times g\left(r+\mu-1, x_{0}(r+\mu-1), \Delta x_{0}(r+\mu-1)\right)\right](\mu+b+1) \frac{\mu-1}{} \\
& +\frac{1}{\Gamma(\mu)} \sum_{r=0}^{b+1}(\mu+b+1-\sigma(r))^{\frac{\mu-1}{2}} g\left(r+\mu-1, x_{0}(r+\mu-1), \Delta x_{0}(r+\mu-1)\right) \\
= & \sum_{k=\mu-1}^{\alpha} x_{0}(k) .
\end{aligned}
$$

Moreover, we have $\Delta_{\mu-2}^{\mu} x_{0}(t)=g\left(t+\mu-1, x_{0}(t+\mu-1), \Delta x_{0}(t+\mu-1)\right)$. This completes the proof.

Some authors tried to find the maximum or exact value of $\sum_{r=0}^{b+1}|G(t, r, \alpha)|$ in some papers (see for example [16, 19] and [17]). Now, we show that $\sum_{r=0}^{b+1} G(t, r, \alpha)$ is bounded, where $G(t, r, \alpha)$ is the Green function of the last result.

Lemma 3.2 For each $t \in \mathbb{N}_{\mu-2}^{b+1+\mu}$ and $\alpha \in \mathbb{N}_{\mu-1}^{b+\mu}$, we have

$$
\left|\sum_{r=0}^{b+1} G(t, r, \alpha)\right| \leq \sum_{r=0}^{b+1}|G(t, r, \alpha)| \leq M_{G}
$$

for some positive number $M_{G}<\infty$. 
Proof Since $\Gamma(\alpha)>0$ for all $\alpha>0$, we have

$$
(\mu+b+1) \stackrel{\mu-1}{=}=\frac{\Gamma(\mu+b+2)}{(b+2) !}>0
$$

for all $\mu>0$ and $b>0$. Thus, $G(t, r, \alpha)$ is a (finite) real number, for all $t \in \mathbb{N}_{\mu-2}^{b+1+\mu}, \alpha \in \mathbb{N}_{\mu-1}^{b+\mu}$ and $r \in \mathbb{N}_{0}^{b+1}$. Consequently, both sums in the statement are finite, because $\mathbb{N}_{0}^{b+1}$ is finite.

Theorem 3.3 Let $g: \mathbb{N}_{\mu-1}^{b+\mu+1} \times \mathbb{R} \times \mathbb{R} \rightarrow \mathbb{R}$ be bounded and continuous in its second and third variables. Then the fractional finite difference equation $\Delta_{\mu-2}^{\mu} x(t)=g(t+\mu-1, x(t+$ $\mu-1), \Delta x(t+\mu-1))$ via the boundary conditions $x(\mu+b+1)=\sum_{k=\mu-1}^{\alpha} x(k)$ and $x(\mu-2)=0$ has a solution $x_{0}$ with $x_{0}(t) \in\left[-M_{G}, M_{G}\right]$, for all admissible $t$.

Proof Since $g$ is bounded, there exists a constant $C$ such that $|g(u, v, w)| \leq C$ for all $u \in$ $\mathbb{N}_{\mu-1}^{b+\mu+1}$ and $v, w \in \mathbb{R}$. Let $\mathcal{X}$ be the Banach space of real valued functions defined on $\mathbb{N}_{\mu-1}^{\mu+b+1}$ via the norm

$$
\|x\|=\max \left\{|x(t)|: t \in \mathbb{N}_{\mu-1}^{\mu+b+1}\right\}
$$

and $\mathcal{K}=\left\{x \in \mathcal{X}:\|x\| \leq C M_{G}\right\}$. One can check easily that $\mathcal{K}$ is a compact, convex, and nonempty subset of $\mathcal{X}$. Now, define the map $T$ on $\mathcal{K}$ by

$$
T x(t)=\sum_{r=0}^{b+1} G(t, r, \alpha) g(r+\mu-1, x(r+\mu-1), \Delta x(r+\mu-1))
$$

for all $t \in \mathbb{N}_{\mu-1}^{\mu+b+1}$. First, we show that $T(\mathcal{K}) \subseteq \mathcal{K}$. Let $x \in \mathcal{K}$ and $t \in \mathbb{N}_{\mu-1}^{\mu+b+1}$. Then

$$
\begin{aligned}
|T x(t)| & =\left|\sum_{r=0}^{b+1} G(t, r, \alpha) g(r+\mu-1, x(r+\mu-1), \Delta x(r+\mu-1))\right| \\
& \leq \sum_{r=0}^{b+1}|G(t, r, \alpha)||g(r+\mu-1, x(r+\mu-1), \Delta x(r+\mu-1))| \\
& \leq C M_{G} .
\end{aligned}
$$

Since $t \in \mathbb{N}_{\mu-2}^{\mu+b+1}$ was arbitrary, $\|T x\| \leq C M_{G}$ and so $T(\mathcal{K}) \subseteq \mathcal{K}$. Now, we show that $T$ is continuous. Let $\epsilon>0$ be given. Since $g$ is continuous in its second and third variables, it is uniformly continuous in its second and third variables on $\left[-\mathrm{CM}_{G}, C M_{G}\right]$ and so there exists $\delta>0$ such that $\left|g\left(t, u_{1}, u_{2}\right)-g\left(t, v_{1}, v_{2}\right)\right|<\frac{\epsilon}{M_{G}}$ for all $t \in \mathbb{N}_{\mu-1}^{\mu+b+1}$ and $u_{1}, u_{2}, v_{1}, v_{2} \in$ $\left[-C M_{G}, C M_{G}\right]$ with $\left|u_{1}-v_{1}\right|<\delta$ and $\left|u_{2}-v_{2}\right|<\delta$. Thus, we get

$$
\begin{aligned}
\mid T y(t) & -T x(t) \mid \\
= & \mid \sum_{r=0}^{b+1} G(t, r, \alpha) g(r+\mu-1, y(r+\mu-1), \Delta y(r+\mu-1)) \\
& \quad-\sum_{r=0}^{b+1} G(t, r, \alpha) g(r+\mu-1, x(r+\mu-1), \Delta x(r+\mu-1)) \mid
\end{aligned}
$$




$$
\begin{aligned}
\leq & \sum_{r=0}^{b+1}|G(t, r, \alpha)| \mid g(r+\mu-1, y(r+\mu-1), \Delta y(r+\mu-1)) \\
& -g(r+\mu-1, x(r+\mu-1), \Delta x(r+\mu-1)) \mid \\
\leq & \sum_{r=0}^{b+1}|G(t, r, \alpha)| \frac{\epsilon}{M_{G}} \leq M_{G} \frac{\epsilon}{M_{G}}=\epsilon
\end{aligned}
$$

for all $t \in \mathbb{N}_{\mu-1}^{\mu+b+1}$. Hence, $\|T x-T y\|<\epsilon$ and so $T$ is continuous on $\mathcal{K}$. By using Theorem 2.2, $T$ has a fixed point $x_{0}$ and so, by using Lemma 3.1, the fractional finite difference equation

$$
\Delta_{\mu-2}^{\mu} x(t)=g(t+\mu-1, x(t+\mu-1), \Delta x(t+\mu-1))
$$

via the boundary conditions $x(\mu+b+1)=\sum_{k=\mu-1}^{\alpha} x(k)$ and $x(\mu-2)=0$ has a solution in $\left[-M_{G}, M_{G}\right]$.

Now, we consider the fractional finite difference equation $\Delta_{\mu-3}^{\mu} x(t)=g(t+\mu-2, x(t+$ $\mu-2)$ ) via the boundary conditions $x(\mu-3)=0, x(\mu+b+1)=0$ and $x(\alpha)=\sum_{k=\gamma}^{\beta} x(k)$, where $2<\mu \leq 3$ and $\alpha, \beta, \gamma \in \mathbb{N}_{\mu-2}^{\mu+b}$ with $\gamma<\beta<\alpha$.

Lemma 3.4 Let $b \in \mathbb{N}_{0}, t \in \mathbb{N}_{0}^{b+3}, 2<\mu \leq 3, g: \mathbb{N}_{\mu-2}^{b+\mu+1} \times \mathbb{R} \rightarrow \mathbb{R}$, and $\alpha, \beta, \gamma \in \mathbb{N}_{\mu-2}^{\mu+b}$ with $\gamma<\beta<\alpha$. Then $x_{0}$ is a solution of the problem $\Delta_{\mu-3}^{\mu} x(t)=g(t+\mu-2, x(t+\mu-2))$ via the boundary conditions $x(\mu+b+1)=0, x(\alpha)=\sum_{k=\gamma}^{\beta} x(k)$, and $x(\mu-3)=0$ if and only if $x_{0}$ is a solution of the fractional sum equation

$$
x(t)=\sum_{r=0}^{b+1} G(t, r, \beta, \alpha) g(r+\mu-2, x(r+\mu-2)),
$$

where

$$
\begin{aligned}
& G(t, r, \beta, \alpha)
\end{aligned}
$$

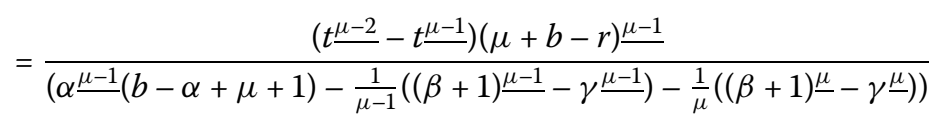

$$
\begin{aligned}
& \times \frac{\left(\frac{1}{\mu-1}\left((\beta+1) \frac{\mu-1}{-}-\gamma^{\frac{\mu-1}{}}\right)(\alpha-\mu+2)-\frac{1}{\mu}\left((\beta+1)^{\underline{\mu}}-\gamma^{\underline{\mu}}\right)\right)}{(b-\alpha+\mu+1)(\mu+b+1) \frac{\mu-2}{} \Gamma(\mu)} \\
& +\frac{\left(t^{\mu-2}(\alpha-\mu+2)-t \frac{\mu-1}{2}\right)(\mu+b-r) \frac{\mu-1}{}}{(b-\alpha+\mu+1)(\mu+b+1) \frac{\mu-2}{2} \Gamma(\mu)} \\
& +\frac{\left(t^{\mu-2}-t^{\mu-1}\right)(\alpha-r-1) \frac{\mu-1}{\mu}}{\left(\alpha \frac{\mu-1}{n}(b-\alpha+\mu+1)-\left(\frac{1}{\mu-1}\left((\beta+1) \frac{\mu-1}{-}-\gamma^{\frac{\mu-1}{}}\right)-\frac{1}{\mu}\left((\beta+1)^{\underline{\mu}}-\gamma^{\underline{\mu}}\right)\right)\right)} \\
& \times \frac{\left(\frac{1}{\mu}\left((\beta+1) \underline{\mu}-\gamma^{\underline{\mu}}\right)-(b+3) \frac{1}{\mu-1}\left((\beta+1) \frac{\mu-1}{\mu}-\gamma^{\underline{\mu-1}}\right)\right)}{\alpha \underline{\mu-1}(b-\alpha+\mu+1) \Gamma(\mu)} \\
& +\frac{\left(t \frac{\mu-1}{\alpha}-(b+3) t \frac{\mu-2}{2}\right)(\alpha-r-1) \frac{\mu-1}{\alpha-1}(b-\alpha+\mu+1) \Gamma(\mu)}{\alpha^{\mu-1}}
\end{aligned}
$$

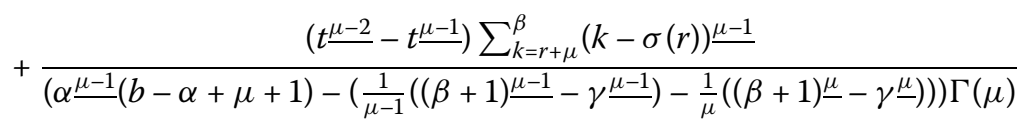

$$
\begin{aligned}
& +\frac{(t-\sigma(r)) \frac{\mu-1}{\Gamma}}{\Gamma(\mu)}
\end{aligned}
$$


whenever $r \leq t-\mu, r \leq \beta-\mu$,

$$
\begin{aligned}
& G(t, r, \beta, \alpha) \\
& =\frac{\left(t^{\underline{\mu-2}}-t^{\frac{\mu-1}{}}\right)(\mu+b-r)^{\underline{\mu-1}}}{\left(\alpha \frac{\mu-1}{\underline{\mu}}(b-\alpha+\mu+1)-\frac{1}{\mu-1}\left((\beta+1) \underline{\mu-1}-\gamma^{\underline{\mu-1}}\right)-\frac{1}{\mu}\left((\beta+1)^{\underline{\mu}}-\gamma^{\underline{\mu}}\right)\right)} \\
& \times \frac{\left(\frac{1}{\mu-1}\left((\beta+1)^{\mu-1}-\gamma^{\underline{\mu-1}}\right)(\alpha-\mu+2)-\frac{1}{\mu}\left((\beta+1)^{\underline{\mu}}-\gamma^{\underline{\mu}}\right)\right)}{(b-\alpha+\mu+1)(\mu+b+1) \frac{\mu-2}{2} \Gamma(\mu)} \\
& +\frac{\left(t \frac{\mu-2}{2}(\alpha-\mu+2)-t \frac{\mu-1}{2}\right)(\mu+b-r) \frac{\mu-1}{\underline{n}}}{(b-\alpha+\mu+1)(\mu+b+1) \frac{\mu-2}{-} \Gamma(\mu)} \\
& +\frac{\left(t^{\underline{\mu-2}}-t^{\underline{\mu-1}}\right)(\alpha-r-1)^{\mu-1}}{\left(\alpha^{\underline{\mu-1}}(b-\alpha+\mu+1)-\left(\frac{1}{\mu-1}\left((\beta+1) \underline{\mu-1}-\gamma^{\underline{\mu-1}}\right)-\frac{1}{\mu}\left((\beta+1)^{\underline{\mu}}-\gamma^{\underline{\mu}}\right)\right)\right)} \\
& \times \frac{\left(\frac{1}{\mu}\left((\beta+1)^{\underline{\mu}}-\gamma^{\underline{\mu}}\right)-(b+3) \frac{1}{\mu-1}\left((\beta+1)^{\mu-1}-\gamma^{\underline{\mu-1}}\right)\right)}{\alpha \underline{\mu-1}(b-\alpha+\mu+1) \Gamma(\mu)} \\
& +\frac{\left(t \frac{\mu-1}{-}-(b+3) t \frac{\mu-2}{2}\right)(\alpha-r-1) \underline{\mu-1}}{\alpha \underline{\mu-1}(b-\alpha+\mu+1) \Gamma(\mu)}
\end{aligned}
$$

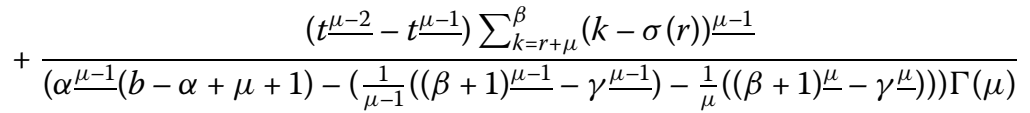

whenever $t-\mu<r, r \leq \beta-\mu$,

$$
\begin{aligned}
& G(t, r, \beta, \alpha) \\
& =\frac{\left(t^{\frac{\mu-2}{}}-t^{\mu-1}\right)(\mu+b-r)^{\mu-1}}{\left(\alpha^{\frac{\mu-1}{}}(b-\alpha+\mu+1)-\frac{1}{\mu-1}\left((\beta+1)^{\underline{\mu-1}}-\gamma^{\underline{\mu-1}}\right)-\frac{1}{\mu}\left((\beta+1)^{\underline{\mu}}-\gamma^{\underline{\mu}}\right)\right)} \\
& \times \frac{\left(\frac{1}{\mu-1}\left((\beta+1) \frac{\mu-1}{\underline{\mu}}-\gamma^{\underline{\mu-1}}\right)(\alpha-\mu+2)-\frac{1}{\mu}\left((\beta+1)^{\underline{\mu}}-\gamma^{\underline{\mu}}\right)\right)}{(b-\alpha+\mu+1)(\mu+b+1) \frac{\mu-2}{2} \Gamma(\mu)} \\
& +\frac{\left(t \frac{\mu-2}{}(\alpha-\mu+2)-t \frac{\mu-1}{2}\right)(\mu+b-r) \frac{\mu-1}{=}}{(b-\alpha+\mu+1)(\mu+b+1) \frac{\mu-2}{} \Gamma(\mu)}
\end{aligned}
$$

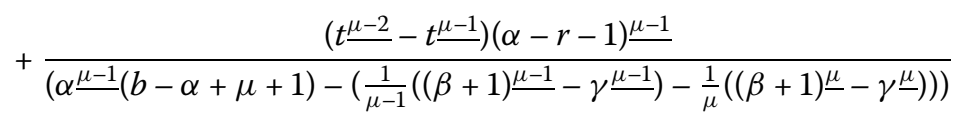

$$
\begin{aligned}
& \times \frac{\left(\frac{1}{\mu}\left((\beta+1)^{\underline{\mu}}-\gamma^{\underline{\mu}}\right)-(b+3) \frac{1}{\mu-1}\left((\beta+1)^{\underline{\mu-1}}-\gamma^{\underline{\mu-1}}\right)\right)}{\alpha^{\underline{\mu-1}}(b-\alpha+\mu+1) \Gamma(\mu)} \\
& +\frac{\left(t \frac{\mu-1}{-}-(b+3) t \frac{\mu-2}{2}\right)(\alpha-r-1) \underline{\mu-1}}{\alpha \underline{\mu-1}(b-\alpha+\mu+1) \Gamma(\mu)}
\end{aligned}
$$

whenever $t-\mu<r, \beta-\mu<r, r \leq \alpha-\mu$,

$$
\begin{aligned}
& G(t, r, \beta, \alpha)
\end{aligned}
$$

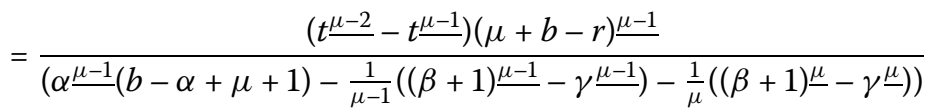

$$
\begin{aligned}
& \times \frac{\left(\frac{1}{\mu-1}\left((\beta+1)^{\mu-1}-\gamma^{\underline{\mu-1}}\right)(\alpha-\mu+2)-\frac{1}{\mu}\left((\beta+1)^{\underline{\mu}}-\gamma^{\underline{\mu}}\right)\right)}{(b-\alpha+\mu+1)(\mu+b+1) \frac{\mu-2}{2} \Gamma(\mu)} \\
& +\frac{\left(t \frac{\mu-2}{2}(\alpha-\mu+2)-t \frac{\mu-1}{=}\right)(\mu+b-r) \underline{\mu-1}}{(b-\alpha+\mu+1)(\mu+b+1) \frac{\mu-2}{\Gamma} \Gamma(\mu)}+\frac{(t-\sigma(r)) \frac{\mu-1}{=}}{\Gamma(\mu)}
\end{aligned}
$$


whenever $r \leq t-\mu, \alpha-\mu<r$ and

$$
\begin{aligned}
& G(t, r, \beta, \alpha) \\
& =\frac{\left(t^{\frac{\mu-2}{2}}-t^{\mu-1}\right)(\mu+b-r) \frac{\mu-1}{}}{\left(\alpha \frac{\mu-1}{\mu}(b-\alpha+\mu+1)-\frac{1}{\mu-1}\left((\beta+1) \frac{\mu-1}{\mu}-\gamma^{\frac{\mu-1}{m}}\right)-\frac{1}{\mu}\left((\beta+1)^{\underline{\mu}}-\gamma^{\underline{\mu}}\right)\right)} \\
& \times \frac{\left(\frac{1}{\mu-1}\left((\beta+1) \frac{\mu-1}{\mu}-\gamma^{\mu-1}\right)(\alpha-\mu+2)-\frac{1}{\mu}\left((\beta+1)^{\underline{\mu}}-\gamma^{\underline{\mu}}\right)\right)}{(b-\alpha+\mu+1)(\mu+b+1) \frac{\mu-2}{} \Gamma(\mu)} \\
& +\frac{\left(t^{\frac{\mu-2}{}}(\alpha-\mu+2)-t \frac{\mu-1}{2}\right)(\mu+b-r) \frac{\mu-1}{}}{(b-\alpha+\mu+1)(\mu+b+1) \frac{\mu-2}{} \Gamma(\mu)}
\end{aligned}
$$

whenever $t-\mu<r, \alpha-\mu<r$.

Proof Let $x_{0}$ be a solution of the problem $\Delta_{\mu-3}^{\mu} x(t)=g(t+\mu-2, x(t+\mu-2))$ via the boundary conditions $x(\mu+b+1)=0, x(\alpha)=\sum_{k=\gamma}^{\beta} x(k)$, and $x(\mu-3)=0$. By using Lemma 2.1, we get

$$
x_{0}(t)=C_{1} t \frac{\mu-1}{+} C_{2} t \frac{\mu-2}{+}+C_{3} t \frac{\mu-3}{\Gamma(\mu)} \sum_{r=0}^{t-\mu}(t-\sigma(r))^{\frac{\mu-1}{}} g\left(r+\mu-2, x_{0}(r+\mu-2)\right) .
$$

Similar to the proof of Lemma 3.1, by using the boundary value conditions we obtain $C_{3}=$ 0 ,

$$
\begin{aligned}
& C_{1}=\frac{-1}{\alpha \frac{\mu-1}{(b-\alpha+\mu+1)}} \sum_{k=\gamma}^{\beta} x_{0}(k) \\
& -\frac{1}{(b-\alpha+\mu+1)(\mu+b+1) \frac{\mu-1}{\frac{\mu}{\Gamma}(\mu)}} \sum_{r=0}^{b+1}(\mu+b-r) \frac{\mu-1}{g} g\left(r+\mu-2, x_{0}(r+\mu-2)\right)
\end{aligned}
$$

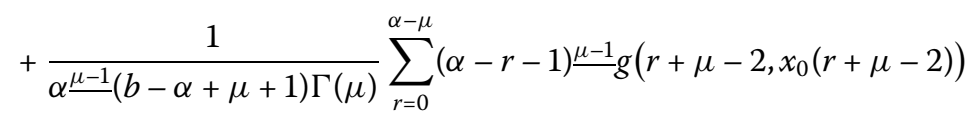

and

$$
\begin{aligned}
& C_{2}=\frac{b+3}{\alpha \frac{\mu-1}{(b-\alpha+\mu+1)}} \sum_{k=\gamma}^{\beta} x_{0}(k) \\
& +\frac{\alpha-\mu+2}{(b-\alpha+\mu+1)(\mu+b+1) \frac{\mu-2}{2} \Gamma(\mu)} \sum_{r=0}^{b+1}(\mu+b-r) \frac{\mu-1}{g} g\left(r+\mu-2, x_{0}(r+\mu-2)\right)
\end{aligned}
$$

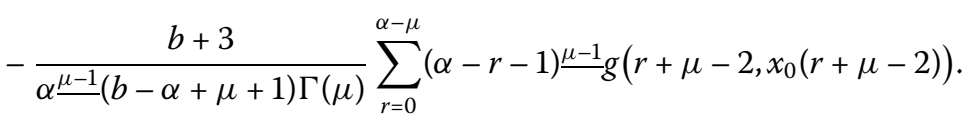

Thus,

$$
\begin{aligned}
x_{0}(t)= & \frac{t \frac{\mu-2}{\alpha \frac{\mu-1}{(}(b-\alpha+\mu+1)} \sum_{k=\gamma}^{\beta-1}}{\beta} x_{0}(k) \\
& +\frac{t \frac{\mu-2}{(b-\alpha+\mu+1)(\mu+b+1) \frac{\mu-2}{} \Gamma(\mu)}}{(b+2)-t_{r=0}^{\mu-1}}(\mu+b-r) \frac{\mu-1}{b+1}
\end{aligned}
$$




$$
\begin{aligned}
& \times g\left(r+\mu-2, x_{0}(r+\mu-2)\right) \\
& +\frac{t \frac{\mu-1}{\alpha}-t \frac{\mu-2}{\underline{\mu}}(b+3)}{(b-\alpha+\mu+1) \Gamma(\mu)} \sum_{r=0}^{\alpha-\mu}(\alpha-r-1) \frac{\mu-1}{} \\
& \times g\left(r+\mu-2, x_{0}(r+\mu-2)\right) \\
& +\frac{1}{\Gamma(\mu)} \sum_{r=0}^{t-\mu}(t-\sigma(r))^{\frac{\mu-1}{g}} g\left(r+\mu-2, x_{0}(r+\mu-2)\right) .
\end{aligned}
$$

To calculate $\sum_{k=\gamma}^{\beta} x_{0}(k)$, by taking the summation $\sum_{k=\gamma}^{\beta}$ on both sides of the above relation gives us

$$
\begin{aligned}
& \sum_{k=\gamma}^{\beta} x_{0}(k)=\frac{\sum_{k=\gamma}^{\beta} k \frac{\mu-2}{\alpha}-\sum_{k=\gamma}^{\beta} k \frac{\mu-1}{\alpha-1}(b-\alpha+\mu+1)}{\alpha_{k=\gamma}^{\beta}} x_{0}(k)
\end{aligned}
$$

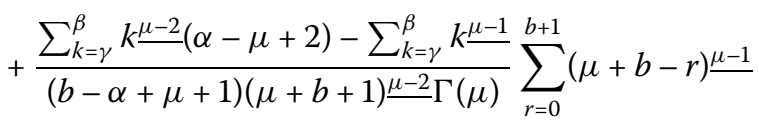

$$
\begin{aligned}
& \times g\left(r+\mu-2, x_{0}(r+\mu-2)\right)
\end{aligned}
$$

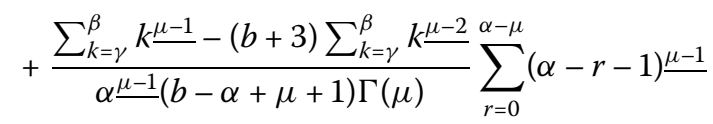

$$
\begin{aligned}
& \times g\left(r+\mu-2, x_{0}(r+\mu-2)\right) \\
& +\frac{1}{\Gamma(\mu)} \sum_{r=0}^{\beta-\mu} \sum_{k=\mu+s}^{\beta}(k-\sigma(r))^{\frac{\mu-1}{g}} g\left(r+\mu-2, x_{0}(r+\mu-2)\right)
\end{aligned}
$$

and so

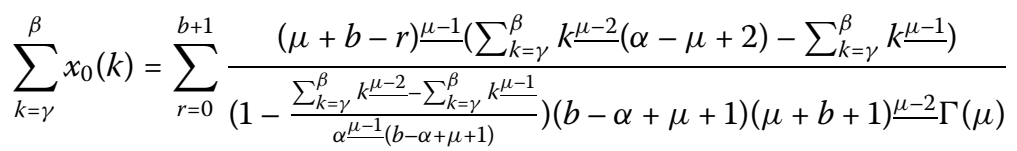

$$
\begin{aligned}
& \times g\left(r+\mu-2, x_{0}(r+\mu-2)\right)
\end{aligned}
$$

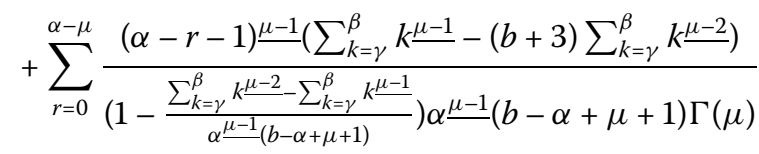

$$
\begin{aligned}
& \times g\left(r+\mu-2, x_{0}(r+\mu-2)\right) \\
& +\sum_{r=0}^{\beta-\mu} \frac{\sum_{k=\mu+s}^{\beta}(k-\sigma(r)) \frac{\mu-1}{\left(1-\frac{\sum_{k=\gamma}^{\beta} k^{\mu-2}-\sum_{k=\gamma}^{\beta} k \frac{\mu-1}{\alpha \underline{\mu-1}}(b-\alpha+\mu+1)}{2}\right)} \Gamma(\mu)}{(\mu)} g\left(r+\mu-2, x_{0}(r+\mu-2)\right) .
\end{aligned}
$$

Since $\sum_{k=\gamma}^{\beta} k^{\underline{\mu-1}}=\sum_{k=\gamma}^{\beta} \Delta_{k}\left(\frac{k^{\underline{\mu}}}{\mu}\right)=\frac{1}{\mu}\left((\beta+1)^{\underline{\mu}}-\gamma^{\underline{\mu}}\right)$ and

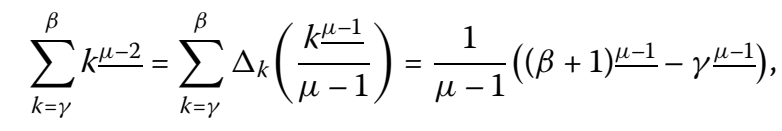


by replacing (3.5) in (3.4), we get

$$
\begin{aligned}
& x_{0}(t) \\
& =\sum_{r=0}^{b+1}\left[\frac{\left(t^{\frac{\mu-2}{2}}-t^{\frac{\mu-1}{2}}\right)(\mu+b-r) \frac{\mu-1}{\mu}}{\left(\alpha^{\frac{\mu-1}{\mu}}(b-\alpha+\mu+1)-\frac{1}{\mu-1}\left((\beta+1)^{\mu-1}-\gamma^{\mu-1}\right)-\frac{1}{\mu}\left((\beta+1)^{\underline{\mu}}-\gamma^{\underline{\mu}}\right)\right)}\right. \\
& \times \frac{\left(\frac{1}{\mu-1}\left((\beta+1) \frac{\mu-1}{-\gamma}-\gamma^{\mu-1}\right)(\alpha-\mu+2)-\frac{1}{\mu}\left((\beta+1) \underline{\mu}-\gamma^{\underline{\mu}}\right)\right)}{(b-\alpha+\mu+1)(\mu+b+1) \frac{\mu-2}{} \Gamma(\mu)} \\
& \left.+\frac{\left(t \frac{\mu-2}{}(\alpha-\mu+2)-t \frac{\mu-1}{n}\right)(\mu+b-r) \frac{\mu-1}{}}{(b-\alpha+\mu+1)(\mu+b+1) \frac{\mu-2}{2} \Gamma(\mu)}\right] g\left(r+\mu-2, x_{0}(r+\mu-2)\right) \\
& +\sum_{r=0}^{\alpha-\mu}\left[\frac{\left(t^{\frac{\mu-2}{2}}-t^{\mu-1}\right)(\alpha-r-1)^{\mu-1}}{\left(\alpha^{\frac{\mu-1}{}}(b-\alpha+\mu+1)-\left(\frac{1}{\mu-1}\left((\beta+1) \frac{\mu-1}{\mu}-\gamma^{\mu-1}\right)-\frac{1}{\mu}\left((\beta+1)^{\mu}-\gamma^{\underline{\mu}}\right)\right)\right)}\right. \\
& \times \frac{\left(\frac{1}{\mu}\left((\beta+1) \underline{\mu}-\gamma^{\underline{\mu}}\right)-(b+3) \frac{1}{\mu-1}\left((\beta+1) \frac{\mu-1}{\mu}-\gamma^{\underline{\mu-1}}\right)\right)}{\alpha^{\underline{\mu-1}}(b-\alpha+\mu+1) \Gamma(\mu)} \\
& \left.+\frac{\left(t \frac{\mu-1}{\alpha}-(b+3) t \frac{\mu-2}{2}\right)(\alpha-r-1) \frac{\mu-1}{2}}{\alpha-\alpha+\mu+1) \Gamma(\mu)}\right] g\left(r+\mu-2, x_{0}(r+\mu-2)\right)
\end{aligned}
$$

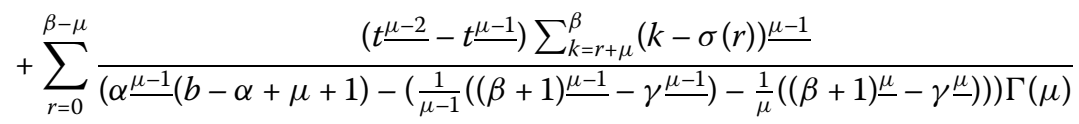

$$
\begin{aligned}
& \times g\left(r+\mu-2, x_{0}(r+\mu-2)\right) \\
& +\sum_{r=0}^{t-\mu} \frac{(t-\sigma(r)) \frac{\mu-1}{\Gamma}}{\Gamma(\mu)} g\left(r+\mu-2, x_{0}(r+\mu-2)\right) \\
& =\sum_{r=0}^{b+1} G(t, r, \beta, \alpha) g\left(r+\mu-2, x_{0}(r+\mu-2)\right) \text {. }
\end{aligned}
$$

Now, let $x_{0}$ be a solution of the fractional sum equation

$$
x(t)=\sum_{r=0}^{b+1} G(t, r, \beta, \alpha) g(r+\mu-2, x(r+\mu-2)) .
$$

Similar to proof of the Lemma 3.1, we conclude that $x_{0}$ is a solution to the problem

$$
\Delta_{\mu-3}^{\mu} x(t)=g(t+\mu-2, x(t+\mu-2))
$$

via the boundary conditions $x(\mu+b+1)=0, x(\alpha)=\sum_{k=\gamma}^{\beta} x(k)$, and $x(\mu-3)=0$. This completes the proof.

By using similar proofs of Lemma 3.2 and Theorem 3.3, we obtain the next results.

Lemma 3.5 For each $t \in \mathbb{N}_{\mu-2}^{b+1+\mu}$ and $\alpha, \beta \in \mathbb{N}_{\mu-2}^{b+\mu}$, we have

$$
\left|\sum_{r=0}^{b+1} G(t, r, \beta, \alpha)\right| \leq \sum_{r=0}^{b+1}|G(t, r, \beta, \alpha)| \leq M_{G}^{\prime}
$$

for some positive number $M_{G}^{\prime}<\infty$. 
Theorem 3.6 Assume that $g: \mathbb{N}_{\mu-2}^{b+\mu+1} \times \mathbb{R} \rightarrow \mathbb{R}$ is continuous and bounded in its second variable. Then the fractional finite difference equation $\Delta_{\mu-3}^{\mu} x(t)=g(t+\mu-2, x(t+\mu-2))$ via the boundary conditions $x(\mu-3)=0, x(\mu+b+1)=0$, and $x(\alpha)=\sum_{k=\gamma}^{\beta} x(k)$ has a solution $x_{0}$ with $x_{0}(t) \in\left[-M_{G}^{\prime}, M_{G}^{\prime}\right]$, for all admissible $t$.

\section{An example}

Now, we provide an example for the first investigated problem.

Example 4.1 Consider the equation

$$
\Delta_{-\frac{2}{3}}^{\frac{4}{3}} x(t)=1+e^{t+\frac{1}{3}}+\sin \left(t+\frac{1}{3}+x\left(t+\frac{1}{3}\right)+\Delta x\left(t+\frac{1}{3}\right)\right)
$$

via the boundary value conditions $x\left(-\frac{2}{3}\right)=0$ and $x\left(\frac{13}{3}\right)=\sum_{k=\frac{1}{3}}^{\frac{4}{3}} x(k)$. We show that this equation has a solution $x_{0}$ with $x_{0}(t) \in[-35.7073,35.7073]$ for all admissible $t$. Let $\mu=\frac{4}{3}$, $\alpha=\frac{4}{3}, b=2$, and

$$
g(u, v, w)=1+e^{u}+\sin (u+v+w)
$$

in the first problem. Thus, we should investigate the fractional finite difference equation

$$
\Delta_{-\frac{2}{3}}^{\frac{4}{3}} x(t)=1+e^{t+\frac{1}{3}}+\sin \left(t+\frac{1}{3}+x\left(t+\frac{1}{3}\right)+\Delta x\left(t+\frac{1}{3}\right)\right)
$$

via the boundary value conditions $x\left(-\frac{2}{3}\right)=0$ and $x\left(\frac{13}{3}\right)=\sum_{k=\frac{1}{3}}^{\frac{4}{3}} x(k)$. Note that the map

$$
g: \mathbb{N}_{\frac{1}{3}}^{\frac{13}{3}} \times \mathbb{R} \times \mathbb{R} \rightarrow \mathbb{R}
$$

is continuous and bounded in its second and third variables. Now, we show that $M_{G}=$ 35.7073. Also, the Green function is given by

$$
\begin{aligned}
G\left(t, r, \frac{4}{3}\right)= & \frac{t^{\frac{1}{3}} \sum_{k=r+\frac{4}{3}}^{\frac{4}{3}}(k-\sigma(r))^{\frac{1}{3}}}{\left(\left(\frac{13}{3}\right)^{\frac{1}{3}}-\frac{3}{4}\left(\frac{7}{3}\right)^{\frac{4}{3}}\right) \Gamma\left(\frac{4}{3}\right)}-\frac{\left(\frac{10}{3}-r\right) \frac{1}{3} \frac{3}{4}\left(\frac{7}{3}\right)^{\frac{4}{3}} t \frac{1}{\frac{1}{3}}}{\left(\frac{13}{3}\right) \frac{\frac{1}{3}}{\frac{3}{3}} \Gamma\left(\frac{4}{3}\right)\left(\left(\frac{13}{3}\right)^{\frac{1}{3}}-\frac{3}{4}\left(\frac{7}{3}\right)^{\frac{4}{3}}\right)} \\
& -\frac{\left(\frac{10}{3}-r\right) \frac{1}{3} t^{\frac{1}{3}}}{\Gamma\left(\frac{4}{3}\right)\left(\frac{13}{3}\right)^{\frac{1}{3}}}+\frac{(t-\sigma(r))^{\frac{1}{3}}}{\Gamma\left(\frac{4}{3}\right)}
\end{aligned}
$$

whenever $r=0, t>\frac{4}{3}$,

$$
G\left(t, r, \frac{4}{3}\right)=-\frac{\left(\frac{10}{3}-r\right)^{\frac{1}{3}} \frac{3}{4}\left(\frac{7}{3}\right)^{\frac{4}{3}} t^{\frac{1}{3}}}{\left(\frac{13}{3}\right) \frac{\frac{1}{3}}{-} \Gamma\left(\frac{4}{3}\right)\left(\left(\frac{13}{3}\right) \frac{\frac{1}{3}}{3}-\frac{3}{4}\left(\frac{7}{3}\right)^{\frac{4}{3}}\right)}-\frac{\left(\frac{10}{3}-r\right)^{\frac{1}{3}} t^{\frac{1}{3}}}{\Gamma\left(\frac{4}{3}\right)\left(\frac{13}{3}\right)^{\frac{1}{3}}}+\frac{(t-\sigma(r))^{\frac{1}{3}}}{\Gamma\left(\frac{4}{3}\right)}
$$

whenever $0<r \leq t-\frac{4}{3}$,

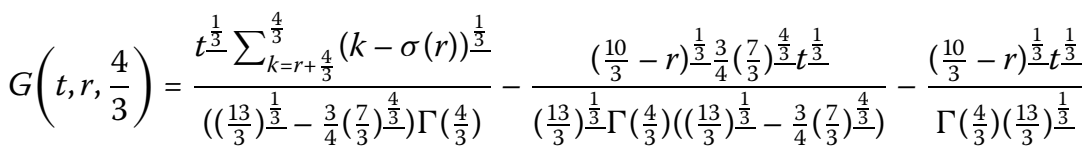


Table 1 The values of the Green function

\begin{tabular}{lrrrr}
\hline $\boldsymbol{t}$ & \multicolumn{1}{c}{$\mathbf{\frac { 4 } { 3 }}$} & \multicolumn{1}{l}{$\frac{\mathbf{7}}{\mathbf{3}}$} & $\frac{\mathbf{1 0}}{\mathbf{3}}$ & $\frac{\mathbf{1 3}}{\mathbf{3}}$ \\
\hline$G\left(t, 0, \frac{4}{3}\right)$ & 1.1089 & 1.7549 & 1.4424 & 1.1697 \\
$G\left(t, 1, \frac{4}{3}\right)$ & -1.1077 & 4.4166 & 4.6063 & 4.7088 \\
$G\left(t, 2, \frac{4}{3}\right)$ & -0.9494 & -1.1077 & 3.8054 & 4.0362 \\
$G\left(t, 3, \frac{4}{3}\right)$ & -0.7121 & -0.8308 & -0.9232 & 3.0272 \\
\hline
\end{tabular}

whenever $t=\frac{4}{3}, r=0$, and

$$
G\left(t, r, \frac{4}{3}\right)=-\frac{\left(\frac{10}{3}-r\right) \frac{\frac{1}{3}}{4} \frac{3}{4}\left(\frac{7}{3}\right)^{\frac{4}{3}} t^{\frac{1}{3}}}{\left(\frac{13}{3}\right) \frac{1}{3} \Gamma\left(\frac{4}{3}\right)\left(\left(\frac{13}{3}\right)^{\frac{1}{3}}-\frac{3}{4}\left(\frac{7}{3}\right) \frac{\frac{4}{3}}{3}\right)}-\frac{\left(\frac{10}{3}-r\right)^{\frac{1}{3}} t^{\frac{1}{3}}}{\Gamma\left(\frac{4}{3}\right)\left(\frac{13}{3}\right)^{\frac{1}{3}}}
$$

for $t-\frac{4}{3}<r$. Thus, for each $r \in \mathbb{N}_{0}^{3}$, one of the values of the Green function $G$ satisfies

$$
\begin{aligned}
& G\left(\frac{4}{3}, 0, \frac{4}{3}\right)=\frac{\left(\frac{1}{3}\right)^{\frac{1}{3}}\left(\frac{4}{3}\right)^{\frac{1}{3}}}{\left(\left(\frac{13}{3}\right)^{\frac{1}{3}}-\frac{3}{4}\left(\frac{7}{3}\right)^{\frac{4}{3}}\right) \Gamma\left(\frac{4}{3}\right)}-\frac{\left(\frac{10}{3}\right)^{\frac{1}{3}} \frac{3}{4}\left(\frac{7}{3}\right)^{\frac{4}{3}}\left(\frac{4}{3}\right)^{\frac{1}{3}}}{\left(\frac{13}{3}\right)^{\frac{1}{3}} \Gamma\left(\frac{4}{3}\right)\left(\left(\frac{13}{3}\right)^{\frac{1}{3}}-\frac{3}{4}\left(\frac{7}{3}\right)^{\frac{4}{3}}\right)}-\frac{\left(\frac{10}{3}\right)^{\frac{1}{3}}\left(\frac{4}{3}\right)^{\frac{1}{3}}}{\Gamma\left(\frac{4}{3}\right)\left(\frac{13}{3}\right)^{\frac{1}{3}}} \\
& =\frac{\Gamma\left(\frac{4}{3}\right) \Gamma\left(\frac{7}{3}\right)}{\left(\frac{\Gamma\left(\frac{16}{3}\right)}{24}-\frac{3}{4} \Gamma\left(\frac{10}{3}\right)\right) \Gamma\left(\frac{4}{3}\right)}-\frac{\frac{3}{4} \frac{\Gamma\left(\frac{13}{3}\right)}{6} \Gamma\left(\frac{10}{3}\right) \Gamma\left(\frac{7}{3}\right)}{\frac{\Gamma\left(\frac{16}{3}\right)}{24}\left(\frac{\Gamma\left(\frac{16}{3}\right)}{24}-\frac{3}{4} \Gamma\left(\frac{10}{3}\right)\right) \Gamma\left(\frac{4}{3}\right)}-\frac{\frac{\Gamma\left(\frac{13}{3}\right)}{6} \Gamma\left(\frac{7}{3}\right)}{\Gamma\left(\frac{4}{3}\right) \frac{\Gamma\left(\frac{16}{3}\right)}{24}} \\
& =1.1089 \text {. }
\end{aligned}
$$

Similar calculations give us the values of $G$ summarized in Table 1.

Thus, $M_{G} \geq \sum_{r=0}^{3}|G(t, r, \alpha)|=35.7073$. Hence by using Theorem 3.6, (4.1) has a solution $x_{0}$ with $x_{0}(t) \in[-35.7073,35.7073]$ for all admissible $t$.

\section{Conclusions}

In this manuscript based on a fixed point theorem we provided the existence results for two fractional finite difference equations in the presence of the sum boundary conditions. One example illustrates our results.

\section{Competing interests}

The authors declare that they have no competing interests.

\section{Authors' contributions}

All authors contributed equally to the writing of this paper. All authors read and approved the final version of the manuscript.

\section{Author details}

${ }^{1}$ Department of Mathematics, Texas A\&M University-Kingsville, 700 University Blvd., Kingsville, TX 78363-8202, USA. ${ }^{2}$ Department of Mathematics, Faculty of Science, King Abdulaziz University, P.O. Box 80204, Jeddah, 21589, Saudi Arabia. ${ }^{3}$ Department of Mathematics, Cankaya University, Ogretmenler Cad. 14, Balgat, Ankara, 06530, Turkey. ${ }^{4}$ Institute of Space Sciences, Magurele, Bucharest, Romania. ${ }^{5}$ Department of Mathematics, Azarbaijan Shahid Madani University, Azarshahr, Tabriz, Iran

\section{Acknowledgements}

Research of the third and fourth authors was supported by Azarbaijan Shahid Madani University.

Received: 16 September 2014 Accepted: 17 October 2014 Published: 31 Oct 2014

\section{References}

1. Baleanu, D, Diethelm, K, Scalas, E, Trujillo, JJ: Fractional Calculus Models and Numerical Methods. World Scientific, Singapore (2012)

2. Kilbas, AA, Srivastava, HM, Trujillo, JJ: Theory and Applications of Fractional Differential Equations. Elsevier, New York (2006) 
3. Podlubny, I: Fractional Differential Equations. Academic Press, San Diego (1999)

4. Atici, FM, Eloe, PW: A transform method in discrete fractional calculus. Int. J. Differ. Equ. 2, 165-176 (2007)

5. Atici, FM, Eloe, PW: Discrete fractional calculus with the nabla operator. Electron. J. Qual. Theory Differ. Equ. 3, special edition I, 1-12 (2009)

6. Baleanu, D, Rezapour, S, Salehi, S: A k-dimensional system of fractional finite difference equations. Abstr. Appl. Anal. 2014, Article ID 312578 (2014)

7. Elaydi, SN: An Introduction to Difference Equations. Springer, Berlin (1996)

8. Goodrich, CS: Some new existence results for fractional difference equations. Int. J. Dyn. Syst. Differ. Equ. 3, 145-162 (2011)

9. Holm, M: Sum and differences compositions in discrete fractional calculus. CUBO 13, 153-184 (2011)

10. Miller, KS, Ross, B: Fractional difference calculus. In: Proc. International Symposium on Univalent Functions, Fractional Calculus and Their Applications. Nihon University, Koriyama (1988)

11. Mohan, JJ, Deekshitulu, GVSR: Fractional order difference equations. Int. J. Differ. Equ. 2012, Article ID 780619 (2012)

12. Pan, Y, Han, Z, Sun, S, Zhao, Y: The existence of solutions to a system of discrete fractional boundary value problems. Abstr. Appl. Anal. 2012, Article ID 707631 (2012)

13. Wu, GC, Baleanu, D: Discrete fractional logistic map and its chaos. Nonlinear Dyn. 75, 283-287 (2014)

14. Wu, GC, Baleanu, D: Discrete chaos in fractional sine and standard maps. Phys. Lett. A 378, 484-487 (2013)

15. Alyousef, K: Boundary value problems for discrete fractional equations. PhD thesis, University of Nebraska-Lincoln (2012)

16. Awasthi, P: Boundary value problems for discrete fractional equations. PhD thesis, University of Nebraska-Lincoln Ann Arbor, MI (2013)

17. Holm, M: The theory of discrete fractional calculus: development and applications. PhD thesis, University of Nebraska-Lincoln, Ann Arbor, MI (2011)

18. Garling, DJH, Gorenstein, D, Diesk, TT, Walters, P: Topics in Metric Fixed Point Theory. Cambridge University Press, Cambridge (1990)

19. Atici, FM, Eloe, PW: Initial value problems in discrete fractional calculus. Proc. Am. Math. Soc. 137, $981-989$ (2009)

10.1186/1687-1847-2014-282

Cite this article as: Agarwal et al.: The existence of solutions for some fractional finite difference equations via sum boundary conditions. Advances in Difference Equations 2014, 2014:282

\section{Submit your manuscript to a SpringerOpen ${ }^{\odot}$ journal and benefit from:}

- Convenient online submission

- Rigorous peer review

- Immediate publication on acceptance

- Open access: articles freely available online

- High visibility within the field

- Retaining the copyright to your article 